\title{
Normal regional brain iron concentration in restless legs syndrome measured by MRI
}

\author{
This article was published in the following Dove Press journal: \\ Nature and Science of Sleep \\ 21 December 2009 \\ Number of times this article has been viewed
}

\author{
Susanne Knake' \\ Johannes T Heverhagen ${ }^{2}$ \\ Katja Menzler' \\ Boris Keil ${ }^{2}$ \\ Wolfgang H Oertel' \\ Karin Stiasny-Kolster ${ }^{1}$ \\ 'Department of Neurology, Center \\ of Nervous Diseases, ${ }^{2}$ Department \\ of Radiology, Philipps University, \\ Marburg, Germany
}

\begin{abstract}
Using a $\mathrm{T}_{2} *$ gradient echo magnetic resonance imaging (MRI) sequence, regional $\mathrm{T}_{2}$ signal intensity (SI) values, a surrogate marker for $\mathrm{T}_{2}$ values, were determined in 12 regions of interest (substantia nigra, pallidum, caudate head, thalamus, occipital white matter, and frontal white matter bilaterally) and in two reference regions (cerebrospinal fluid and bone) in 12 patients suffering from moderate to severe idiopathic restless legs syndrome (RLS; mean age $58.5 \pm 8.7$ years) for $12.1 \pm 9.1$ years and in 12 healthy control subjects (mean age $56.8 \pm$ 10.6 years). Iron deposits shorten $\mathrm{T}_{2}$ relaxation times on $\mathrm{T}_{2}$-weighted MRI. We used regional $\mathrm{T}_{2}{ }^{*}$ SI to estimate regional $\mathrm{T}_{2}$-values. $\mathrm{A}_{2}$-change ratio was calculated for each region of interest relative to the reference regions. We did not find significant differences in any of the investigated brain regions. In addition, serum measures involved in iron metabolism did not correlate with $\mathrm{T}_{2}$ SI values. We could not replicate earlier findings describing reduced regional brain iron concentrations in patients with RLS. Our results do not support the view of substantially impaired regional brain iron in RLS.
\end{abstract}

Keywords: restless legs syndrome, pathophysiology, iron, MRI, substantia nigra

\section{Introduction}

Iron insufficiency has been proposed to play a central role in restless legs syndrome (RLS) pathophysiology indicated primarily by the secondary causes of RLS such as pure iron deficiency anemia and end stage renal disease or pregnancy which are both associated with altered iron metabolism. It has also been shown that frequent blood donors have an increased risk of RLS. ${ }^{1}$ Overall, it has gained wide clinical acceptance that RLS is commonly occurring with iron deficiency anemia, although there have been few studies documenting this relationship. ${ }^{2-4}$ In addition, it has been shown that RLS severity negatively correlates with serum ferritin. ${ }^{3,5}$ Correcting iron status in RLS patients with iron deficiency has been found to reduce RLS symptoms. ${ }^{3,6}$ Complete remission of RLS symptoms has also been reported after intravenous substitution of iron in RLS patients with and without clear anemia. ${ }^{7-10}$ Thus pharmacological data as well as data from secondary RLS support a relation between iron deficiency and RLS. For evaluating the iron status of RLS patients, serum and cerebrospinal fluid (CSF) measures involved in iron metabolism, ${ }^{11}$ autopsy evaluation of brain iron status, ${ }^{12,13}$ transcranial parenchymal ultrasound, ${ }^{14,15}$ and magnetic resonance imaging (MRI) measurements ${ }^{16,17}$ have been performed. All of these data supported the view that there is a measurable brain iron deficiency particularly in the substantia nigra in RLS patients. ${ }^{18} \mathrm{~T}_{2}$-hypointensity is thought to represent iron deposition as iron deposits shorten $\mathrm{T}_{2}$ relaxation times on $\mathrm{T}_{2}$-weighted MRIs. ${ }^{19}$ Using a cranial MRI
Correspondence: Susanne Knake Department of Neurology, Rudolf-Bultmann-Strasse 8, 35033 Marburg, Germany $\mathrm{Tel}+49642$ I5865200 Fax +49642 I5865208 Email knake@staff.uni-marburg.de submit your manuscript $\mid$ www.dovepress.com

Dovepress 
scan with a standard $\mathrm{T}_{2}{ }^{*}$-sequence, differences of regional mean $\mathrm{T}_{2}$-values can be measured throughout the brain as a consequence of different brain iron concentrations. Up to now, significant differences in brain iron concentrations were found in the substantia nigra in RLS patients as compared to controls in two studies using $\mathrm{T}_{2}{ }^{*}$-weighted MRI. ${ }^{16,17}$ In this study we investigated regional brain iron concentrations using a standard $\mathrm{T}_{2}{ }^{*}$-sequence but failed to replicate earlier findings of reduced brain iron in the substantia nigra in patients with RLS compared to healthy controls.

\section{Patients and methods}

Twelve patients (nine women, three men, mean age $58.5 \pm 8.7$ years, range $43-46$ years) with idiopathic RLS for $12.1 \pm 9.1$ years (range $2-30$ years) were included. Five patients had early-onset RLS with symptom onset before the age of 45 years. All patients met the standardized diagnostic criteria of the International Restless Legs Syndrome Study Group, ${ }^{8}$ and neurological examination revealed no evidence of additional neurological or psychiatric disease. Eight patients were treated with dopaminergic agents for RLS (levodopa, $\mathrm{n}=5$; pramipexole, $\mathrm{n}=3$ ). The mean overall duration of treatment with dopaminergics (including previous medication) in these patients was $3.4 \pm 2.4$ years (range $1-9$ years). Four patients were untreated and never received dopaminergics. RLS severity was assessed considering the overall status when not taking the current medication (if applicable) by means of the Clinical Global Impression (CGI; $1=$ normal to $6=$ very severe). CGI ratings revealed that six patients suffered from moderate RLS $(\mathrm{CGI}=4)$ and six patients from severe RLS $(\mathrm{CGI}=5)$. Four patients had a positive family history. Controls consisted of 12 gender- and age-matched subjects (mean age $56.8 \pm 10.6$ years, range $41-74$ years) in whom RLS was clinically excluded. RLS patients had the following blood iron values: iron, $16.7 \pm 3.3 \mu \mathrm{mol} / \mathrm{L}$ (range 12.4 to 21.1 ); ferritin, $111.9 \pm 63.4 \mu \mathrm{g} / \mathrm{L}$ (range 22 to 193 ); transferrin, $2.5 \pm 0.4 \mathrm{~g} / \mathrm{L}$ (range 1.9 to 3.2 ); transferrin saturation, $26.7 \% \pm 8.9 \%$ (range 20 to 44 ); soluble transferrin receptor, $3.3 \pm 1.0 \mathrm{mg} / \mathrm{L}$ (range 2 to 5 ). All values were within the normal range and did not significantly differ from control subjects who had the following mean values: iron, $18.7 \pm 6.6 \mu \mathrm{mol} / \mathrm{L}$ (range 8.5 to 27.1 ); ferritin, $124.7 \pm 171.8 \mu \mathrm{g} / \mathrm{L}$ (range 7 to 614 ); transferrin, $2.4 \pm 0.4 \mathrm{~g} / \mathrm{L}$ (range 2 to 3.2 ); transferrin saturation, $31.1 \% \pm 11.3 \%$ (range 12 to 46 ); soluble transferrin receptor, $3.1 \pm 0.8 \mathrm{mg} / \mathrm{L}$ (range 2.1 to 4.6 ).

All subjects were scanned using a whole head $\mathrm{T}_{2} *$ weighted gradient echo sequence (TR $800 \mathrm{~ms}$, TE $26 \mathrm{~ms}, \mathrm{FA}: 20^{\circ}$, FOV $230 \times 230 \mathrm{~mm}^{2}$, Matrix $256 \times 192$, slice thickness $5 \mathrm{~mm}$ ) on a Siemens 1.5T Sonata Scanner (Siemens Medical Solutions, Erlangen, Germany). Average $\mathrm{T}_{2}{ }^{*}$ signal intensity (SI) values were determined in selected regions of interest. Regions of interest (ROIs) were placed in the following areas in each hemisphere: the substantia nigra, the pallidum, the caudate head, the thalamus as well as the occipital white matter and the frontal white matter bilaterally. All ROIs were placed using a standardized placement procedure utilizing atlasbased rules with morphological landmarks in each individual participant's volumes. All ROIs were placed by the same rater who was blinded to the subjects status (patient or control) and were created as a sphere with a diameter of $2 \mathrm{~mm}^{3}$ (totalling a number of 100 isotropic voxels) to avoid arbitrary sizes of ROIs across participants, and to avoid inclusion of multiple white matter bundles in regional measurements. The average $\mathrm{T}_{2}$ SI value and its standard deviation were determined for each ROI and for two reference regions of interest. One of these reference ROIs was placed in the middle of the ventricles, measuring CSF, the other was placed at the scull measuring the $\mathrm{T}_{2}$ SI value of the bone. SI was measured as a surrogate marker for $\mathrm{T}_{2}$-values. In order to provide objective measurements the $\mathrm{T}_{2}$-change ratio $\left(\mathrm{T}_{2} \mathrm{CR}\right)$ was calculated as followed: $\mathrm{T}_{2} \mathrm{CR}=(\mathrm{SI}[\mathrm{ROI}]-\mathrm{SI}$ Reference $) / \mathrm{SI}$ Reference. Differences in $\mathrm{T}_{2} \mathrm{CR}$ between patients and control subjects were analyzed using the Mann-Whitney $U$ Test. For correlation analysis, Spearman rank correlation coefficients were calculated. Results are reported as mean \pm standard deviation. The significance level was set at $\alpha=5 \%$ with adjustment for multiple testing by the Bonferroni method.

The study was performed in accordance with the Declaration of Helsinki and approved by the local ethics committee. Written informed consent was given by all participants.

\section{Results}

The $\mathrm{T}_{2} \mathrm{CR}$ values did not significantly differ between RLS patients and controls for any of the investigated brain regions both for the CSF and bone ratios (see Tables 1 and 2).

In RLS patients there was no significant correlation between brain iron values and RLS severity or CGI scores. In both groups we did not find a significant correlation between $\mathrm{T}_{2} \mathrm{CR}$ values (CSF and bone ratios) and age or sex. In addition, serum iron, ferritin, transferrin, transferrin saturation and the soluble transferrin receptor concentration did not correlate with brain $\mathrm{T}_{2} \mathrm{CR}$ values (CSF and bone ratios) in both groups. Subanalysis of untreated and treated patients did not reveal significant differences in $\mathrm{T}_{2} \mathrm{CR}$ values (CSF and bone ratios) and we found no significant differences between early-onset and late-onset RLS. 
Table I Mean $\mathrm{T}_{2} \mathrm{CR}$ values (bone ratio) in various brain regions of RLS patients and control subjects

\begin{tabular}{llll}
\hline & RLS & Controls & P value \\
\hline Substantia nigra & & & \\
Right & $0.53 \pm 0.26$ & $0.39 \pm 0.14$ & 0.133 \\
Left & $0.47 \pm 0.26$ & $0.37 \pm 0.13$ & 0.272 \\
Pallidum & & & \\
Right & $0.52 \pm 0.34$ & $0.43 \pm 0.21$ & 0.686 \\
Left & $0.55 \pm 0.28$ & $0.40 \pm 0.20$ & 0.418 \\
Caudate head & & & \\
Right & $0.94 \pm 0.36$ & $0.81 \pm 0.11$ & 0.563 \\
Left & $0.86 \pm 0.32$ & $0.81 \pm 0.12$ & 0.795 \\
Thalamus & & & \\
Right & $0.33 \pm 0.06$ & $0.31 \pm 0.05$ & 0.401 \\
Left & $0.91 \pm 0.33$ & $0.86 \pm 0.10$ & 0.644 \\
Occipital WM & & & \\
Right & $0.59 \pm 0.32$ & $0.57 \pm 0.19$ & 0.840 \\
Left & $0.68 \pm 0.36$ & $0.54 \pm 0.14$ & 0.148 \\
Frontal WM & & & \\
Right & $0.40 \pm 0.39$ & $0.47 \pm 0.10$ & 0.795 \\
Left & $0.54 \pm 0.27$ & $0.44 \pm 0.09$ & 0.773 \\
\hline Absrevitions & & & \\
\hline
\end{tabular}

Abbreviations: RLS, restless legs syndrome; $T_{2} C R, T_{2}$-change ratio; WM, white matter.

\section{Discussion}

In this study we did not find significant abnormalities in brain iron content of RLS patients in any of the investigated brain regions such as the substantia nigra, pallidum, caudate head, or thalamus. Serum measures involved in iron metabolism such as serum iron, ferritin, transferrin, transferrin saturation, and soluble transferrin receptor did not correlate with $\mathrm{T}_{2}$ CR-values.

In an earlier study Allen and colleagues ${ }^{16}$ investigated five RLS patients with a similar MRI method and reported decreased iron concentration in the substantia nigra and the putamen compared to five age- and sex-matched controls. In a subsequent study they performed MRI in a considerable larger sample including 22 early-onset RLS patients, 19 late-onset RLS patients, and 39 controls. In that study they found a significant lower iron index in the substantia nigra only in early-onset RLS but not in late-onset compared to controls. ${ }^{17}$ These results are consistent with CSF studies of the same group showing iron insufficiency in CSF predominantly in early-onset RLS. ${ }^{11}$ In agreement, autopsy evaluations of brain iron status which were all performed in early-onset RLS showed a brain iron deficit in the substantia nigra. ${ }^{12,13}$ In addition, transcranial ultrasound studies revealed a significantly reduced hyperechogenicity (hypoechogenicity) in the substantia nigra in RLS patients when compared with normal control subjects which is supposed to indicate reduced regional iron concentration. ${ }^{14,15}$ All of these data supported the view that there is a measurable brain iron deficiency particularly in the substantia nigra in RLS patients. In contrast, a recent MRI study investigating patients with RLS with significant hypoechogenicity in the substantia nigra in the transcranial ultrasound found no significant differences in $T_{2}$ values reflecting iron concentration in the substantia nigra, ${ }^{20} \mathrm{a}$ finding that is also supported by the present study. However, Godau and colleauge ${ }^{20}$ found MR signal changes in the caudate nucleus, the thalamus, and the red nucleus, which could not be confirmed in the present and earlier studies. ${ }^{21}$

In all of the studies describing an iron deficiency in the substantia nigra, patients were either on dopaminergic medication or pretreated with dopaminergics and taken off medication for the investigation. Therefore it was discussed that dopaminergic medication could possibly have affected brain iron concentration ${ }^{16}$ and thus the results of above mentioned studies.

Using a similar technique, ${ }^{19}$ we could not replicate the MRI findings of earlier studies ${ }^{16,17}$ and did not find a regional

Table 2 Mean $\mathrm{T}_{2} \mathrm{CR}$ values (CSF ratio) in various brain regions of RLS patients and control subjects

\begin{tabular}{llll}
\hline & RLS & Controls & P value \\
\hline Substantia nigra & & & \\
Right & $-0.45 \pm 0.08$ & $-0.48 \pm 0.06$ & 0.298 \\
Left & $-0.48 \pm 0.08$ & $-0.48 \pm 0.06$ & 0.707 \\
Pallidum & & & \\
Right & $-0.46 \pm 0.07$ & $-0.47 \pm 0.04$ & 0.685 \\
Left & $-0.45 \pm 0.06$ & $-0.48 \pm 0.06$ & 0.140 \\
Caudate head & & & \\
Right & $-0.31 \pm 0.06$ & $-0.32 \pm 0.05$ & 0.563 \\
Left & $-0.34 \pm 0.07$ & $-0.32 \pm 0.04$ & 0.885 \\
Thalamus & & & \\
Right & $-0.33 \pm 0.06$ & $-0.31 \pm 0.05$ & 0.401 \\
Left & $-0.32 \pm 0.04$ & $-0.30 \pm 0.04$ & 0.353 \\
Occipital WM & & & \\
Right & $-0.43 \pm 0.06$ & $-0.42 \pm 0.05$ & 0.727 \\
Left & $-0.40 \pm 0.06$ & $-0.43 \pm 0.04$ & 0.602 \\
Frontal WM & & & \\
Right & $-0.48 \pm 0.15$ & $-0.45 \pm 0.04$ & 0.539 \\
Left & $-0.45 \pm 0.05$ & $-0.48 \pm 0.15$ & $0.56 \mathrm{I}$ \\
\hline
\end{tabular}

Abbreviations: CSF, cerebrospinal fluid; RLS, restless legs syndrome; $T_{2} C R, T_{2}$-change ratio; WM, white matter. 
brain iron deficit neither in early- or in late-onset RLS. Since we also investigated some patients who were on dopaminergic medication it is unlikely that the RLS-specific medication accounts for the different results. Discrepancies might have arisen as $\mathrm{T}_{2}$-values are not only affected by iron concentration but also by water content of the brain. Therefore, correlation with volumetric measures should be performed in future.

Since iron increases in the brain with aging, ${ }^{22}$ age differences could be a confounding factor. However, we and others $^{16,17}$ found no correlation between brain iron content and age. One possible reason for the different results might be differences in serum iron variables which were either not assessed in the previous MRI studies ${ }^{16}$ or only used for exclusion of patients with iron deficiency (serum ferritin $<18 \mu \mathrm{g} / \mathrm{L}$, iron saturation $<16 \%$ ). ${ }^{17}$ Therefore it is possible that patients in the previous MRI studies had lower iron values than our patients or lower compared to their control group resulting in significant differences. However, we did not find a significant correlation between $\mathrm{T}_{2}$-values and serum iron parameters.

Another possible explanation for the different MR findings concerning the iron concentration in the substantia nigra and various other brain regions might be the different MR sequences used in the different studies which may be influenced by different aspects of tissue iron composition ${ }^{20}$ und thus lead to different results.

Further studies with larger sample sizes, assessment of iron variables, all patients off RLS medication and consistent MR sequences are necessary to better clarify the role of brain iron in RLS. In addition, studies using more sophisticated methods which can be applied in vivo to study untreated patients and which are able to detect also small quantitative differences in brain iron, eg, moving to higher field strengths or the use of new imaging methods such as three-dimensional (3D) gradient echo imaging or susceptibility-weighted imaging ${ }^{19}$ should be performed. Despite the limitations in the MRI measurements of brain iron, our results do not support the view of substantially impaired regional brain iron in RLS.

\section{Disclosures}

The authors report no conflicts of interest in this work.

\section{References}

1. Silber MH, Richardson JW. Multiple blood donations associated with iron deficiency in patients with restless legs syndrome. Mayo Clin Proc. 2003;78:52-54.

2. Ekbom KA. Restless legs syndrome. Acta Med Scand. 1945;158:4-122.

3. O'Keeffe ST, Gavin K, Lavan JN. Iron status and restless legs syndrome in the elderly. Age Ageing. 1994;23:200-203.

4. Rangarajan S, D'Souza GA. Restless legs syndrome in Indian patients having iron deficiency anemia in a tertiary care hospital. Sleep Med. 2007;8:247-251.

5. Sun ER, Chen CA, Ho G, Earley CJ, Allen RP. Iron and the restless legs syndrome. Sleep. 1998;21:371-377.

6. Davis BJ, Rajput A, Rajput ML, Aul EA, Eichhorn GR. A randomized, double-blind, placebo-controlled trial of iron in restless legs syndrome. Eur Neurol. 2000;43:70-75.

7. Norlander NB. Therapy in restless legs. Acta Med Scand. 1953;145: 453-457.

8. Norlander NB. Restless legs. Br J Phys Med. 1954;17:160-162.

9. Earley CJ, Heckler D, Allen RP. The treatment of restless legs syndrome with intravenous iron dextran. Sleep Med. 2004;5:231-235.

10. Sloand JA, Shelly MA, Feigin A, Bernstein P, Monk RD. A doubleblind, placebo-controlled trial of intravenous iron dextran therapy in patients with ESRD and restless legs syndrome. Am J Kidney Dis. 2004:43:663-670.

11. Earley C, Connor JR, Beard JL, Malecki EA, Epstein DK, Allen RP. Abnormalities in CSF concentrations of ferritin and transferrin in restless legs syndrome. Neurology. 2000;54:1698-1700.

12. Connor JR, Boyer PJ, Menzies SL, et al. Neuropathological examination suggests impaired brain iron acquisition in restless legs syndrome. Neurology. 2003;61:304-309.

13. Connor JR, Wang XS, Patton SM, et al. Decreased transferrin receptor expression by neuromelanin cells in restless legs syndrome. Neurology. 2004;62:1563-1567.

14. Schmidauer C, Sojer M, Seppi K, et al. Transcranial ultrasound shows nigral hypoechogenicity in restless legs syndrome. Ann Neurol. 2005;58:630-634.

15. Godau J, Schweitzer KJ, Liepelt I, Gerloff C, Berg D. Substantia nigra hypoechogenicity: definition and findings in restless legs syndrome. Mov Disord. 2007;22:187-192.

16. Allen RP, Barker PB, Wehrl F, Song HK, Earley CJ. MRI measurement of brain iron in patients with restless legs syndrome. Neurology. 2001;56:263-265.

17. Early C, Barker PB, Horska A, Allen RP. MRI-determined regional brain iron concentrations in early- and late-onset restless legs syndrome. Sleep Med. 2006;7:458-461.

18. Allen RP, Earley CJ. The role of iron in restless legs syndrome. Mov Disord. 2007;22(Suppl 18):S440-S448.

19. Haacke M, Cheng NY, House MJ, et al. Imaging iron stores in the brain using magnetic resonance imaging. Magn Reson Imaging. 2005;23:1-25.

20. Godau J, Klose U, Di Santo A, Schweitzer K, Berg D. Multiregional brain iron deficiency in restless legs syndrome. Mov Disord. 2008;23:1184-1187.

21. Etgen T, Draganski B, Ilg C, et al. Bilateral thalamic gray matter changes in patients with restless legs syndrome. Neuroimage. 2005;24:1242-1247.

22. Hallgreen B, Sourrander P. The effect of age on the non-haemin iron in the human brain. J Neurochem. 1958;3:41-51.
Nature and Science of Sleep

\section{Publish your work in this journal}

Nature and Science of Sleep is an international, peer-reviewed, open access journal covering all aspects of sleep science and sleep medicine, including the neurophysiology and functions of sleep, the genetics of sleep, sleep and society, biological rhythms, dreaming, sleep disorders and therapy, and strategies to optimize healthy sleep. The journal welcomes

\section{Dovepress}

original research, clinical \& epidemiological studies, reviews \& evaluations, case reports and extended reports. The manuscript management system is completely online and includes a very quick and fair peerreview system, which is all easy to use. Visit http://www.dovepress.com/ testimonials.php to read real quotes from published authors. 